\title{
Abnormal thyroid hormone metabolism in mice lacking the monocarboxylate transporter 8
}

\author{
Marija Trajkovic, ${ }^{1}$ Theo J. Visser, ${ }^{2}$ Jens Mittag, ${ }^{3}$ Sigrun Horn, ${ }^{1}$ Jan Lukas, ${ }^{1}$ Veerle M. Darras, ${ }^{4}$ \\ Genadij Raivich, ${ }^{5}$ Karl Bauer, ${ }^{3}$ and Heike Heuer ${ }^{1}$ \\ ${ }^{1}$ Leibniz Institute for Age Research/Fritz Lipmann Institute, Jena, Germany. ${ }^{2}$ Department of Internal Medicine, \\ Erasmus Medical Center, Rotterdam, The Netherlands. ${ }^{3}$ Max Planck Institute for Experimental Endocrinology, Hannover, Germany. \\ ${ }^{4}$ Laboratory of Comparative Endocrinology, Katholieke Universiteit Leuven, Leuven, Belgium. ${ }^{5}$ Perinatal Brain Repair Group, \\ University College London, London, United Kingdom.
}

\begin{abstract}
In humans, inactivating mutations in the gene of the thyroid hormone transporter monocarboxylate transporter $8(M C T 8 ; S L C 16 A 2)$ lead to severe forms of psychomotor retardation combined with imbalanced thyroid hormone serum levels. The MCT8-null mice described here, however, developed without overt deficits but also exhibited distorted 3,5,3'-triiodothyronine (T3) and thyroxine (T4) serum levels, resulting in increased hepatic activity of type 1 deiodinase (D1). In the mutants' brains, entry of T4 was not affected, but uptake of T3 was diminished. Moreover, the T4 and T3 content in the brain of MCT8-null mice was decreased, the activity of D2 was increased, and D3 activity was decreased, indicating the hypothyroid state of this tissue. In the CNS, analysis of T3 target genes revealed that in the mutants, the neuronal T3 uptake was impaired in an area-specific manner, with strongly elevated thyrotropin-releasing hormone transcript levels in the hypothalamic paraventricular nucleus and slightly decreased RC3 mRNA expression in striatal neurons; however, cerebellar Purkinje cells appeared unaffected, since they did not exhibit dendritic outgrowth defects and responded normally to T3 treatment in vitro. In conclusion, the circulating thyroid hormone levels of MCT8-null mice closely resemble those of humans with MCT8 mutations, yet in the mice, CNS development is only partially affected.
\end{abstract}

\section{Introduction}

Thyroid hormone is essential for the metabolic homeostasis of almost all tissues. Even more importantly, it is crucially involved in the maturation and function of the CNS (1-3). Absence of thyroid hormone during pre- and early postnatal periods affects a variety of processes, such as neuronal migration and differentiation as well as myelination, thus leading to a vast array of clinical manifestations $(4,5)$. In humans, fetal and/or neonatal thyroid hormone deficiency results in the syndrome of cretinism, a disorder characterized by severe mental retardation, neurological deficits (e.g., movement disorders, spasticity, and speech problems), as well as hearing impairment.

Actions of thyroid hormone are predominantly mediated by the binding of 3,5,3'-triiodothyronine (T3) to its nuclear receptors, thereby regulating gene expression (6). T3, the principal bioactive form of thyroid hormone, is produced by outer ring deiodination of the prohormone thyroxine (T4), a reaction catalyzed by type 1 deiodinase (D1) and D2. Inactivation of T4 and T3 is accomplished predominantly by D3, which exhibits inner ring deiodinase activity $(7,8)$. Since deiodination and T3-dependent gene transcription take place intracellularly, thyroid hormone has to cross cell membranes in order to be metabolized and to interact with its receptors.

Nonstandard abbreviations used: D1, type 1 deiodinase; GPD2, $\alpha$-glycerol-3-phosphate dehydrogenase; ISH, in situ hybridization; MCT8, monocarboxylate transporter 8; MMI, 2-mercapto-1-methyl-imidazol; OATP, organic anion-transporting polypeptide; PVN, paraventricular hypothalamic nucleus; T3, 3,5,3'-triiodothyronine; T4, thyroxine; TRH, thyrotropin-releasing hormone; TSH, thyroid-stimulating hormone.

Conflict of interest: The authors have declared that no conflict of interest exists. Citation for this article: J. Clin. Invest. 117:627-635 (2007). doi:10.1172/JCI28253.
A vast body of evidence has accumulated to suggest that the passage of thyroid hormone does not occur via passive diffusion but is rather facilitated by transporter proteins (9). Among several iodothyronine transporters that display different kinetics and substrate preferences, the monocarboxylate transporter 8 (MCT8) deserves special interest, since it has been characterized as being one of the most specific and active thyroid hormone transporters identified so far (10). The physiological importance of MCT8 as a thyroid hormone transporter was demonstrated by the identification of patients in about 20 different families carrying mutations or deletions in the MCT8 gene, which is localized on the X chromosome (11-13). The syndrome of psychomotor retardation diagnosed in these patients affects children from an early age and consists of severe developmental delay and neurological damage, including lack of speech development, profound proximal hypotonia with poor head control, spastic quadriplegia, and dystonic movement. In addition to the psychomotor retardation, patients with MCT8 mutations present abnormal serum thyroid hormone parameters, in particular highly elevated serum T3 levels and decreased T4 concentrations in the presence of normal thyroid-stimulating hormone (TSH) levels.

The mechanisms leading to this combination of abnormal thyroid hormone levels and severe neurological deficits are not yet understood. Since MCT8 is already expressed in murine neurons during embryonic development (14), impaired neuronal T3 uptake and metabolism during critical periods of brain development is thought to contribute to the pathogenesis of this syndrome. In order to elucidate the underlying mechanisms, we were interested in analyzing genetically modified mice rendered MCT8 deficient by targeted disruption of the MCT8 (Slc16a2; solute carrier family 16, member 2) gene. Here, we provide a description of these animals. 
A

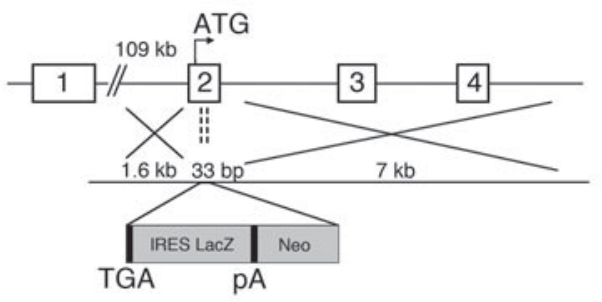

B

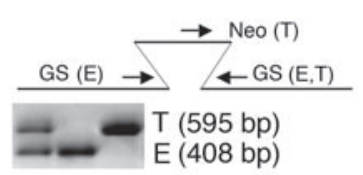

E

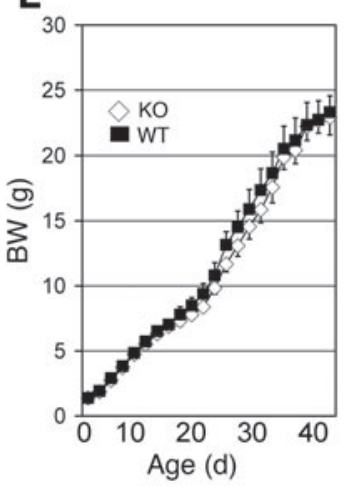

C
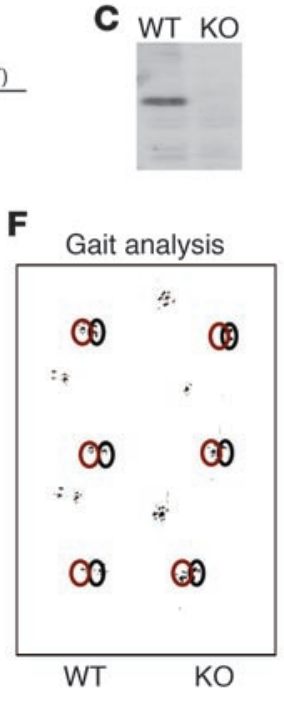

F Gait analysis
D W
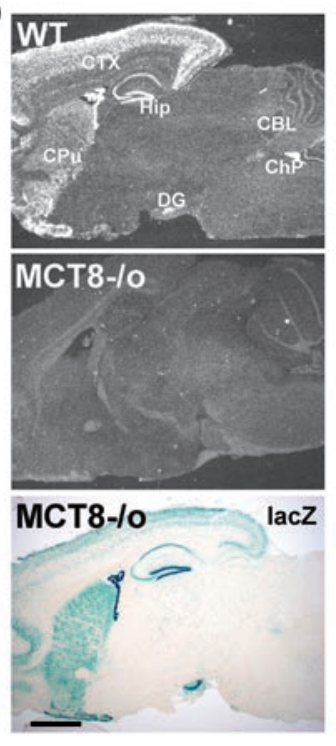

Figure 1

Generation and analysis of MCT8-null mice. (A) Targeting strategy for the MCT8-knockout mouse. As described in Methods, 33 bp of exon 2 were replaced by a lacZ/neomycin reporter cassette. pA, polyadenylation signal; TGA, stop codon. (B) Homologous recombination was confirmed by PCR analysis using 3 primers recognizing the endogenous $(E)$ and the targeted $(T)$ allele as indicated and described in Methods. GS, gene-specific primer; Neo, neomycin cassette-specific primer. (C) Absence of MCT8 protein in MCT8-deficient animals was demonstrated by Western blot analysis of liver homogenates. (D) Deletion of the MCT8 gene was further confirmed by radioactive ISH demonstrating that MCT8 mRNA expression in sagittal brain sections of wild-type animals is completely diminished in sections derived from MCT8-null mice. Deletion of the MCT8 gene was also demonstrated by the expression of lacZ in MCT8-10 animals. CBL, cerebellum; ChP, choroid plexus; $\mathrm{CPu}$, caudate-putamen; CTX, cerebral cortex; DG, dentate gyrus; Hip, hippocampus. Scale bar in $\mathbf{D}: 1.5 \mathrm{~mm}$. (E) MCT8-null mice develop normally, as indicated by the growth curve. (F) Gait analysis did not reveal any signs of ataxia.

\section{Results}

Generation and phenotypical analysis of MCT8-null mice. Female $M C T 8^{+/-}$mice were generated by targeted homologous recombination, whereby a 33-bp fragment of exon 2 was replaced by a lacZ neomycin reporter cassette (Figure 1A). Genotyping was performed by PCR analysis as described in Methods and illustrated in Figure 1B. For propagation, $M C T 8^{+/-}$females were mated with wild-type C57BL/N6 and NMRI males in order to obtain MCT8-null animals on 2 different genetic backgrounds. In agreement with the localization of the MCT8 gene on the X chromosome, PCR analysis revealed that $50 \%$ of the male offspring exhibited an $M C T 8^{-/ 0}$ genotype. After $\mathrm{MCT}^{-/ o}$ males and $\mathrm{MCT}^{+/-}$females were mated, MCT8-null pups were born at the expected Mendelian frequency excluding prenatal mortality of mutant animals. The disruption of the MCT8 gene was further validated in MCT8-null mice by the absence of MCT8 protein in liver homogenates as detected by Western blot analysis (Figure 1C), the absence of MCT8 transcripts as revealed by in situ hybridization (ISH) histochemistry, as well as by the complementary expression of $\beta$-galactosidase (Figure 1D).

Surprisingly, MCT8-null mice developed normally independent of the genetic background and were indistinguishable from their wild-type or heterozygous littermates. We could not observe any significant differences in growth rate among the genotypes (Figure 1E), and phenotypically, there were no overt deficits, even with regard to motor functions. As indicated by the results of footprint analysis, there was also no indication of ataxia (Figure 1F). MCT8null mice of both sexes were fertile and reproduced normally. While microcephaly has been observed in patients with MCT8 mutations $(11,13,15)$, we did not detect any differences in the brain weight of MCT8-null (0.466 $\pm 0.027 \mathrm{~g})$ and control animals $(0.478 \pm 0.028 \mathrm{~g})$ as assessed at the age of 6 weeks.
Serum iodothyronine levels in the MCT8-null versus control mice. MCT8-1o males and their wild-type littermates on an NMRI background were analyzed at P21 with regard to their thyroidal state (Figure 2). In MCT8-null mice, mean serum T4 levels were reduced to $34 \%$ of mean control values. In contrast, the mean serum T3 level was increased to $205 \%$ of mean control values, resulting in a 6.3 -fold increase in the T3/T4 ratio in the MCT8-null animals. Thus, MCT8-null mice exhibit the same abnormal serum thyroid hormone parameters as diagnosed in patients with MCT8 mutations.

Expression of T3-responsive genes in the liver. As expected from the high serum T3 levels of MCT8-null mice, D1 activities in the liver were found to be increased 2-fold compared with those of wild-type littermates (Figure 3A). A similar strong increase in D1 mRNA expression throughout the liver of MCT8-null mice was also demonstrated by ISH analysis (Figure 3B). Furthermore, we performed quantitative real-time PCR analysis to determine not
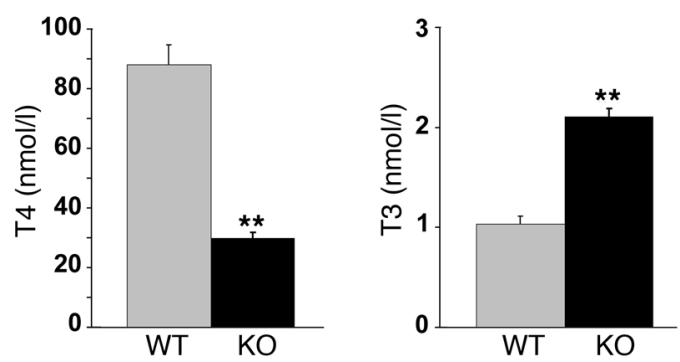

\section{Figure 2}

Serum thyroid hormone levels in 21-day-old male MCT8-null mice and wild-type littermates. Per group, 12 animals were analyzed. Bars represent the mean \pm SEM of values obtained in each group. ${ }^{* *} P<0.0001$. 
A
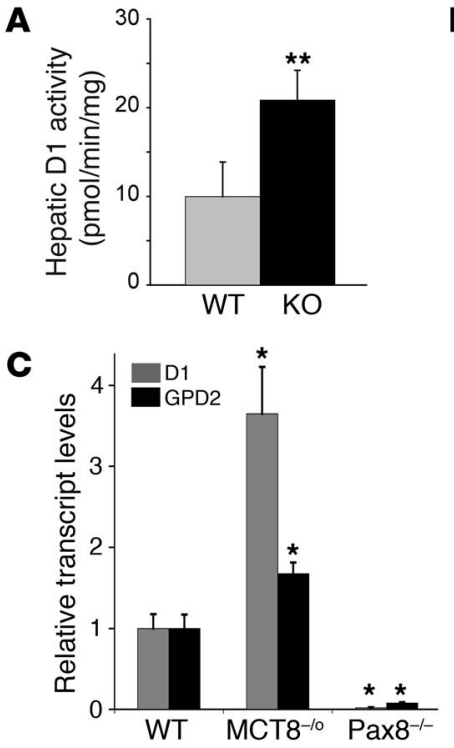

D

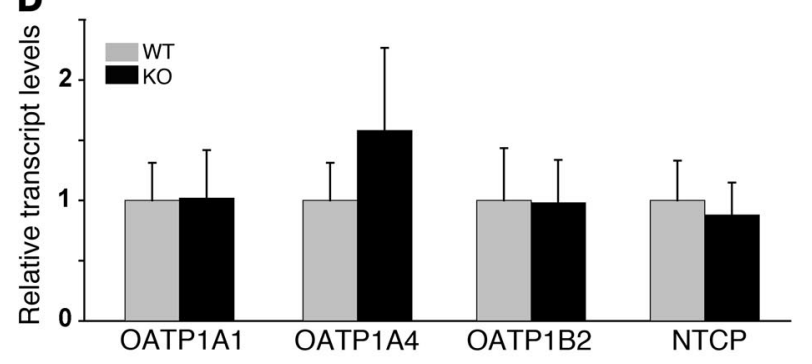

only $D 1$ expression levels but also the relative transcript levels of the hepatic T3-responsive gene encoding $\alpha$-glycerol-3-phosphate dehydrogenase (GPD2) (16). In addition, we included $\mathrm{Pax}^{-/-}$mice in our studies in order to compare MCT8-null animals with mice that are born without a functional thyroid gland and are, therefore, completely athyroid (17). As illustrated in Figure 3C, the transcript levels of D1 and GPD2 were both found to be elevated in the MCT8-null mice compared with wild-type littermates, whereas in hypothyroid $\mathrm{Pax}^{-/-}$animals, these 2 T3-regulated genes were downregulated. These observations indicate that in response to the markedly increased serum T3 levels, the liver of MCT8-deficient animals is in a hyperthyroid state despite the absence of MCT8. In search of compensatory mechanisms, we also analyzed by real-time PCR the hepatic transcript levels of the organic aniontransporting polypeptides OATP1A1, OATP1A4, and OATP1B2 and the sodium/taurocholate cotransporting polypeptide NTCP, which are known to transport thyroid hormones with some efficiency (18). Since we did not observe compensatory alterations in

\section{Figure 4}

Entry of ${ }^{125}$-labeled iodothyronines into the brain and liver of MCT8null mice and wild-type littermates. Adult animals (4 per experiment) were s.c. injected with $2 \times 10^{6} \mathrm{cpm}\left[{ }^{125} \mid\right] \mathrm{T} 4$ or [ $\left.{ }^{125}\right]$ T 3 . At indicated time points, blood samples were taken and the radioactivity was determined. Subsequently, the animals were perfused with saline. Liver and brain were then removed and weighed, and the radioactivity was determined. Results are presented as the percentage of injected dose normalized to the amount of tissue (in $\mathrm{ml}$ or $\mathrm{g}$ ). ${ }^{*} P<0.05$.

\section{Figure 3}

Analysis of D1 expression in the liver of MCT8-null and wild-type animals. (A) D1 enzymatic activity was determined in liver and kidney preparations from 21-day-old MCT8-null mice and wild-type littermates. (B) ISH analysis of hepatic MCT8 and D1 expression in wild-type mice and D1 expression in MCT8-null animals. In MCT8-null mice, expression of $D 1$ in liver was highly upregulated compared with the expression of $D 1$ in liver of control animals. Expression of MCT8 in wild-type animals is shown in the lower panel. Scale bar: $450 \mu \mathrm{m}$. (C) Hepatic D1 and GPD2 mRNA levels were determined in 21-day-old MCT8-null mice, wild-type littermates, and athyroid $\mathrm{Pax} 8^{-/-}$mice by real-time PCR. Bars in $\mathbf{A}$ and $\mathbf{C}$ represent the mean \pm SEM of values obtained in each group. ${ }^{\star} P<0.05 ;{ }^{* *} P<0.0001$. (D) Transcript levels of putative hepatic thyroid hormone transporters were examined in the liver of MCT8-null mice and controls by real-time PCR. This analysis revealed no statistical differences in the expression levels for the organic anion transporting polypeptides OATP1A1, OATP1A4, and OATP1B2 and the $\mathrm{Na}^{+} /$taurocholate-cotransporting polypeptide NTCP between the different genotypes.

the hepatic transcript levels of these candidates (Figure 3D), we were interested in studying the contribution of MCT8 in mediating thyroid hormone transport processes by analyzing the uptake of radiolabeled T4 and T3 into various tissues of MCT8-deficient mice and wild-type control animals.

Uptake of radiolabeled thyroid hormones into the liver and brain of MCT8-null versus control mice. After s.c. injection of ${ }^{125}$ I-labeled T4 or ${ }^{125}$ I-labeled T3 into adult MCT8-null males and wild-type littermates ( 4 animals per time point and genotype), the amount of radioactivity in blood and liver samples was determined at indicated time points. As shown in Figure 4, the radioactivity in the blood samples rapidly increased, reaching, for $\mathrm{T} 4$, a peak value of approximately $3 \%-4 \%$ of the injected dose and for T3, $1 \%-1.5 \%$ of the injected dose. No significant differences were noted in blood samples derived from MCT8-null mice or wild-type littermates, indicating that the absence of MCT8 did not affect the clearance rate of T4 or T3. Moreover, following the injection of $\left[{ }^{125} \mathrm{I}\right] \mathrm{T} 4$ or $\left[{ }^{125} \mathrm{I}\right] \mathrm{T} 3$, the total amount of radioactivity accumulating in the
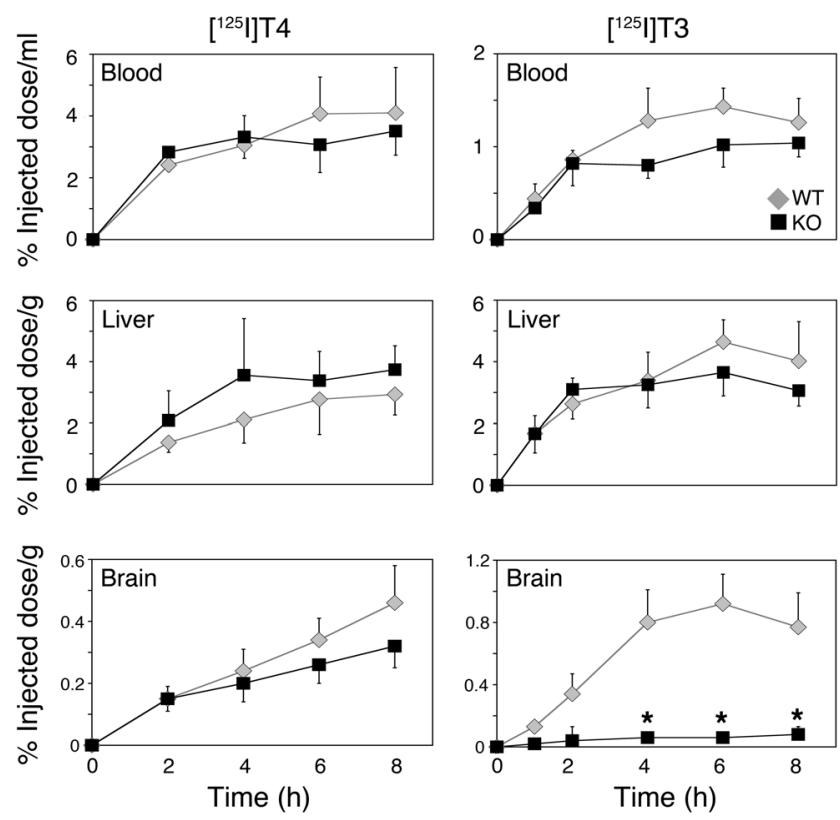
A

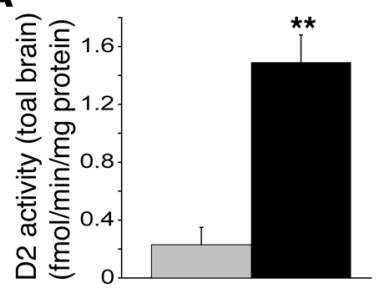

B

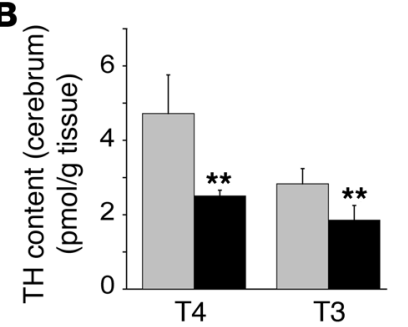

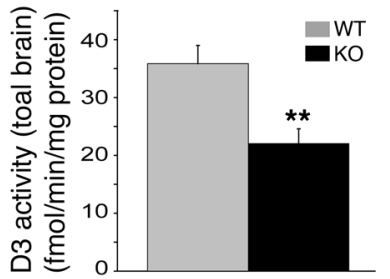

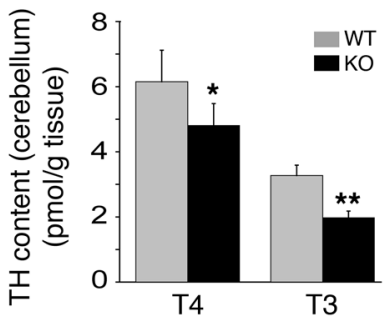

liver increased similarly in MCT8-deficient animals and wild-type controls. Uptake of [ $\left.{ }^{125} \mathrm{I}\right] \mathrm{T} 4$ into the brain was also not affected in MCT8-null mice. In contrast, uptake of [ $\left.{ }^{125} \mathrm{I}\right] \mathrm{T} 3$ into the brain of MCT8-null mice was severely impaired. Whereas in the brain of control mice, the amount of $\left[{ }^{125} \mathrm{I}\right] \mathrm{T} 3$ reached a peak value of $1 \%$ of the injected dose after 6 hours, $\left[{ }^{125} \mathrm{I}\right] \mathrm{T} 3$ content in the brain of MCT8-null mice was only slightly above background levels at all time points analyzed. These findings provide evidence for a significant contribution of MCT8 as a thyroid hormone transporter facilitating the entry of $\mathrm{T} 3$ into the brain.

Analysis of thyroid hormone metabolism in the CNS. In view of the neurological deficits of patients with MCT8 mutations as well as the impaired uptake of $\left[{ }^{125} \mathrm{I}\right] \mathrm{T} 3$ into the brain of MCT8-null mice, it was most interesting to study the thyroid hormone status and metabolism in the CNS. Analysis of D2 activities revealed a 7-fold upregulation in the brain of MCT8-null mice compared with control littermates, while D3 activities were significantly decreased to $62 \%$ of control values (Figure 5A). Since D2 is negatively and D3 is positively regulated by thyroid hormone, these changes most likely reflect a compensatory mechanism to cope with low serum T4 concentrations. Indeed, T4 content in the cerebrum and cerebellum of MCT8-null mice was found to be decreased to $53 \%$ and $78 \%$ of control values, whereas T3 levels were reduced to $61 \%$ and $66 \%$, respectively (Figure $5 \mathrm{~B}$ ). These findings suggest that increased local T3 production by increased D2 activity and decreased T3 breakdown by decreased D3 activity can only partially compensate for the low T4 levels.

T3 responsiveness of MCT8-deficient Purkinje cells. In addition to choroid plexus structures, tanycytes, and larger capillary endothelial cells, expression of MCT8 has also been localized to certain neuronal cell populations (14), where it might be critically involved in T3 uptake. We analyzed the development of the cerebellum,

\section{Figure 5}

Analysis of the thyroid state in the brain of MCT8-null mice. (A) Compared with those in wild-type littermates, brain D2 activities are increased in MCT8-null mice, whereas D3 activities are decreased. Bars represent the mean \pm SEM of values obtained in each group. (B) Determination of the tissue thyroid hormone content revealed decreased T4 and T3 levels in the cerebrum and cerebellum of MCT8null mice, respectively. Bars represent the mean $\pm S D$ of values obtained in each group. ${ }^{*} P<0.05 ;{ }^{* *} P<0.005$.

a brain area highly dependent on proper thyroid hormone supply, in MCT8-null mice. In line with the absence of any gross motor deficits, histological analysis did not reveal any signs of abnormal cerebellar differentiation. Purkinje cells that express MCT8 as revealed by ISH in wild-type controls (14) and by lacZ staining in the MCT8-null animals (Supplemental Figure 1A; supplemental material available online with this article; doi:10.1172/JCI28253DS1) exhibited normal dendritic development as assessed by calbindin staining of vibratome sections derived from MCT8-null mice at P12 (Figure 6, A and C). Furthermore, the maturation of the external granule cell layer was neither delayed nor accelerated (Figure $6, \mathrm{~B}$ and D), indicating the presence of sufficient amounts of thyroid hormones during the first postnatal weeks. To substantiate the notion that T3-dependent Purkinje cell development is not affected by MCT8 deficiency, we analyzed the T3 responsiveness of MCT8-deficient Purkinje cells in vitro under defined T3 concentrations. For that purpose, pooled cerebellar samples were prepared from neonatal MCT8-null or wild-type mice and cultured under serum-free conditions in either the presence or in the absence of $1 \mathrm{nM} \mathrm{T} 3$ for 14 days. Calbindin staining of these cultures and quantification of dendritic outgrowth revealed that MCT8-deficient Purkinje cells (Figure 6, E and F) respond to T3 treatment with the same increase in dendritic parameters $(2.89 \pm 0.67$-fold $)$ as wildtype animals (3.01 \pm 0.72 -fold) (Figure 6, G and H).

Thyroidal status in striatal and bypothalamic neurons. In addition to studying cerebellar Purkinje cells, we aimed to assess the intracellular T3 status in MCT8-deficient neurons by studying the
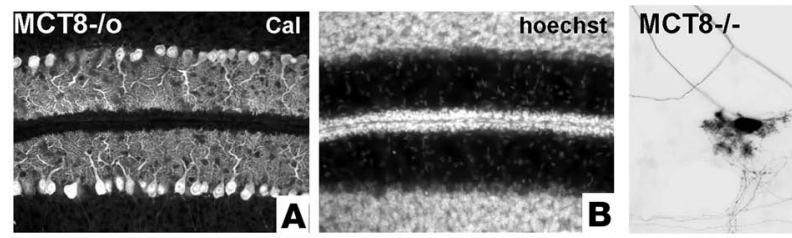

$-\mathrm{T3}$
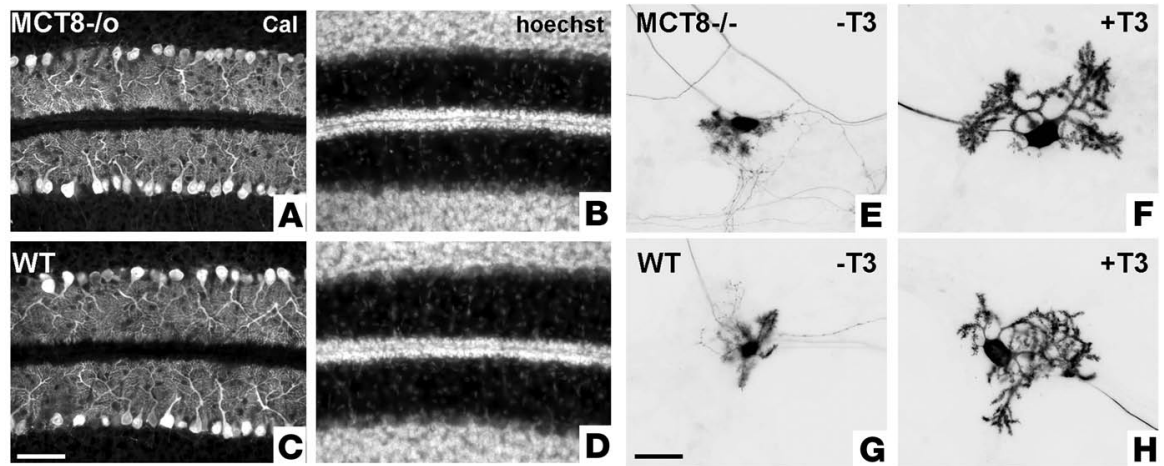

WT

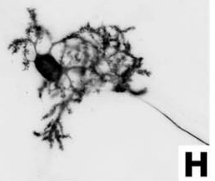

\section{Figure 6}

Cerebellar analysis of MCT8-null mice. Calbindin (Cal) (A and $\mathbf{C})$ and Hoechst (B and $\mathbf{D})$ staining of cerebellar vibratome sections from 12-day-old MCT8-null mice (A and $\mathbf{B}$ ) and wild-type controls (C and $\mathbf{D})$ did not reveal any differences with regard to the development of Purkinje cells ( $\mathbf{A}$ and C) or the maturation of the external granule cell layer (B and D). When cultured without T3 as described in Methods, Purkinje cells from MCT8-null mice (E) and wild-type controls (G) showed severely impaired development. In the presence of $1 \mathrm{nM}$ T3, the dendritic development of cultured Purkinje cells derived from MCT8 mutants $(\mathbf{F})$ was stimulated just as strongly as that of Purkinje cells from wild-type controls (H). Scale bar in C (for $\mathbf{A}-\mathbf{D}$ ): $100 \mu \mathrm{m}$; in $\mathbf{G}$ (for $\mathbf{E}-\mathbf{H}$ ): $50 \mu \mathrm{m}$. 

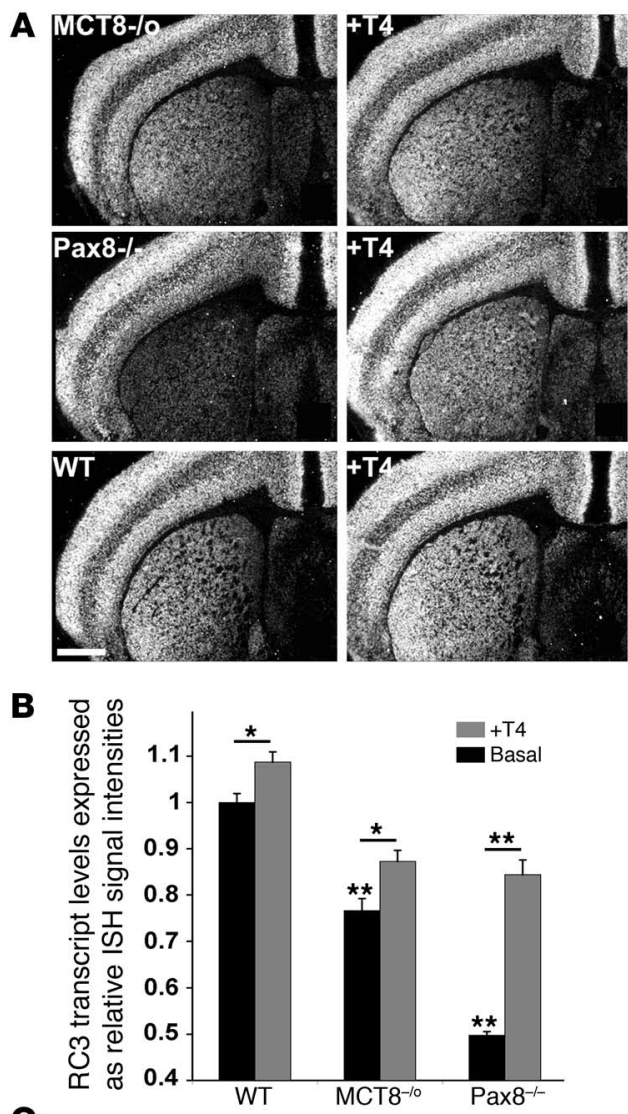

C
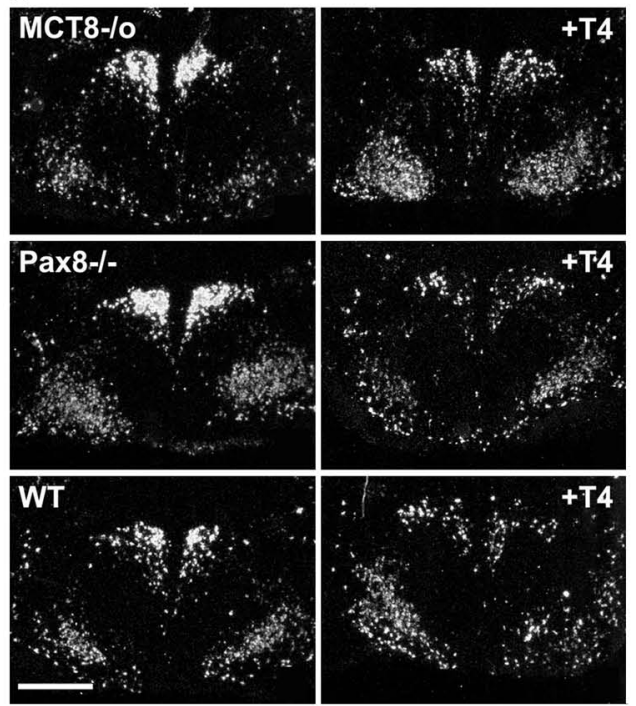

expression levels of T3-sensitive genes in the brain of untreated MCT8-null mice as well as mutants injected with T4 (200 ng/g BW) daily between P18 and P21. This treatment resulted in an increase in serum T4 levels from $26.8 \pm 2.8$ to $68.6 \pm 4.4 \mathrm{nmol} / \mathrm{l}$ and in an increase of serum T3 levels from $2.63 \pm 0.26$ to $5.49 \pm 0.97 \mathrm{nmol} / 1$ in MCT8-null mice.

$R C 3$ expression in the striatum. As a first approach, we analyzed the transcript levels of RC3/neurogranin in the striatum, an MCT8expressing brain area (Supplemental Figure 1B) critically involved in motor control. In the striatum, $R C 3$ has been shown to be positively

\section{Figure 7}

Expression of the T3-regulated genes $R C 3$ and $T R H$ in the striatum and in the PVN. (A) Compared with that in wild-type controls, RC3 mRNA expression in the striatum was modestly decreased in MCT8null mice and strongly in athyroid $\mathrm{Pax} 8^{-/-}$mice. After treatment with T4 (200 ng/g BW for 3 consecutive days), striatal RC3 mRNA levels increased in MCT8 mutants and in $\mathrm{Pax}^{-/-}$mice as well as in control animals. Scale bar: $1.5 \mathrm{~mm}$. (B) The evaluation was validated by NIH image analysis of the hybridization signals. ${ }^{*} P<0.05$; ${ }^{* *} P<0.0001$. (C) In hypothalamic PVN neurons, ISH analysis revealed highly upregulated $T R H$ mRNA levels in MCT8-null mice and athyroid $P a x 8^{-1-}$ mice compared with control animals. After treatment with T4 (200 ng/g BW for 3 consecutive days), TRH mRNA expression was strongly reduced in wild-type animals as well as in MCT8 mutants and Pax $8^{-1-}$ mice. Scale bar: $450 \mu \mathrm{m}$.

regulated by T3 (19). In this brain region, we observed a lateromedial gradient in the $R C 3$ expression pattern in the presence as well as in the absence of MCT8 (Figure 7A). ISH analysis also revealed a decrease in striatal signal intensities for RC3 in MCT8-null mice compared with wild-type controls, whereas in athyroid $\mathrm{Pax} 8^{-/-}$ mice (included for comparison), RC3 expression in the striatum was very low, as expected. Densitometric analysis (Figure 7B) confirmed that striatal $R C 3 \mathrm{mRNA}$ expression is significantly decreased in MCT8-null animals to $76 \%$ and in athyroid $\mathrm{Pax} 8^{-/-}$ mice to $50 \%$ of wild-type values. Treatment of animals for 3 days with T4 (200 ng/g BW) increased striatal $R C 3 \mathrm{mRNA}$ expression in the athyroid $\mathrm{Pax} 8^{-/-}$animals as well as in MCT8-null mice (Figure $7 \mathrm{~A}$, right panel). However, control values were not reached during this short period of T4 treatment (Figure 7B).

Thyrotropin-releasing hormone expression in bypothalamic paraventricular neurons. We next determined the transcript levels of another interesting target gene of thyroid hormone action, thyrotropin-releasing hormone (TRH), in the paraventricular hypothalamic nucleus (PVN), where, in agreement with our previous studies, MCT8 was expressed as visualized by lacZ staining of MCT8-deficient PVN neurons (Supplemental Figure 1C). Within the negative-feedback loop of the hypothalamus-pituitary-thyroid (HPT) axis, TRH mRNA expression in the PVN is inversely correlated to thyroid hormone levels and thus represents a suitable sensor of intracellular T3 content.

Compared with those of wild-type animals, TRH transcript levels were highly upregulated in PVN neurons of MCT8-null mice, similar in magnitude to the transcript levels found in the athyroid $\mathrm{Pax} 8^{-/-}$ mouse (Figure 7C, left panels). In animals made hyperthyroid by injection of a supraphysiological dose of T4 for 3 days, TRH expression in the PVN was not only downregulated in control animals and in $\mathrm{Pax}^{-/-}$mice but also in MCT8-null animals (Figure 7C, right panels), indicating that even in the absence of MCT8, TRH-positive PVN neurons are still capable of responding to T4 treatment.

Analysis of thyroid hormone metabolism in the pituitary. The elevated TRH mRNA levels in the PVN of MCT8-null mice prompted us to analyze whether other components of the HPT axis were affected as well. D2 activities in pituitaries of MCT8-null mice were found to be moderately increased, by a factor of 1.5 , indicating a mild hypothyroid situation (Supplemental Figure 2A). However, determination of serum thyroid-stimulating hormone (TSH) levels did not reveal any statistically significant difference between MCT8-null mice and wild-type controls (Supplemental Figure 2B). Furthermore, 
A

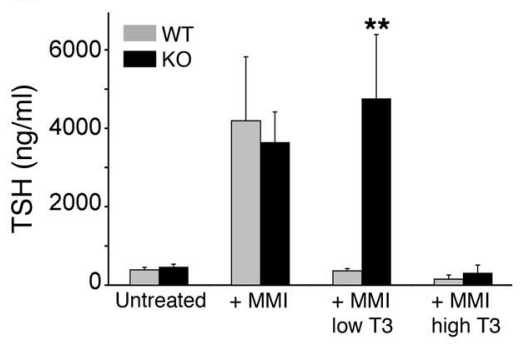

B

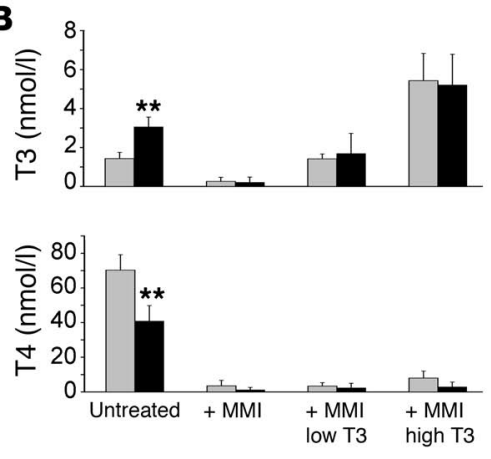

c
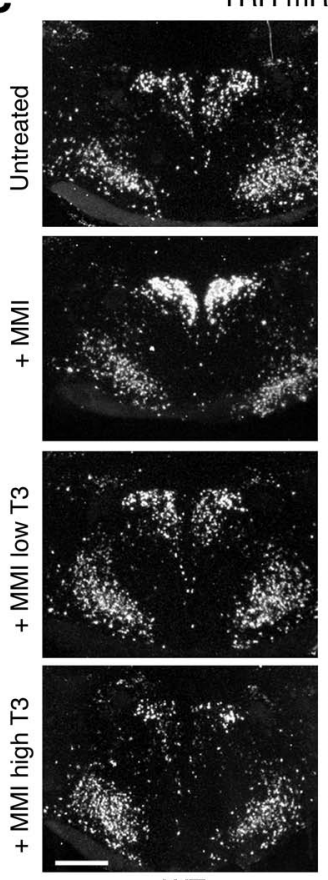

WT
TRH mRNA level
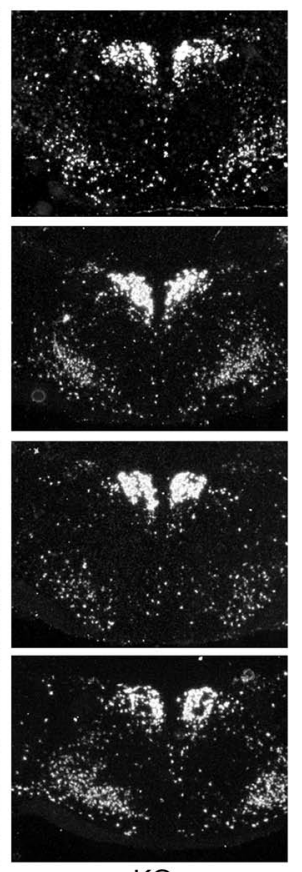

$\mathrm{KO}$

\section{Figure 8}

Effect of the thyroid state on serum TSH, T3, and T4 levels and hypothalamic TRH mRNA levels in MCT8-null mice and wild-type littermates. Adult MCT8-null mice and wild-type littermates were rendered hypothyroid by $\mathrm{MMl} /$ perchlorate treatment for 12-14 weeks as described in Methods. As a consequence, serum TSH levels $(\mathbf{A})$ and ISH signal intensities reflecting $T R H$ transcript levels $(\mathbf{C})$ were markedly increased in animals of both genotypes, whereas serum T3 and T4 levels (B) were close to the detection limit. In wild-type animals, s.c. injection of a low dose of T3 ( $5 \mathrm{ng} / \mathrm{g} \mathrm{BW}$ for 3 consecutive days) was sufficient to normalize not only serum T3 and TSH levels but also to restore to normal TRH mRNA levels in the PVN, whereas in MCT8-null animals, neither TSH nor TRH expression was found to be suppressed in the presence of normal serum T3 concentrations. Only rendering the MCT8-null mice hyperthyroid by injecting a high dose of T3 ( $25 \mathrm{ng} / \mathrm{g}$ BW for 3 days) resulted in a downregulation of serum TSH levels, whereas $T R H$ expression in the PVN was only slightly reduced. Scale bar: $330 \mu \mathrm{m} .{ }^{* *} P<0.0001$. neither mRNA expression levels of TSH nor the transcript levels of other thyroid hormone target genes in the pituitary (growth hormone [GH], TRH-R1, D2) were affected by the absence of MCT8 as assessed by real-time PCR analysis (Supplemental Figure 2C).

These results suggest that the pituitary of MCT8-null mice does not adequately perceive the high serum $\mathrm{T} 3$ levels but rather exhibits a partial resistance to T3. In order to test this hypothesis, adult MCT8-null mice and their wild-type littermates were rendered hypothyroid by treatment with 2-mercapto-1-methyl-imidazol/ perchlorate (MMI/perchlorate) for 12-14 weeks. As illustrated in Figure 8, this treatment resulted in a strong decrease in T3 and $\mathrm{T} 4$ serum levels in wild-type animals and mutants. Consequently, serum TSH levels were upregulated 8- to 10-fold in animals of both genotypes (Figure 8A). Additionally, TRH expression in the PVN was found to be highly upregulated in wild-type mice (Figure $8 \mathrm{C}$ ) and appeared to be upregulated even more strongly in the PVN of MMI-treated MCT8-null mice. Injecting hypothyroid wild-type mice for 3 days with a low dose of T3 (5 ng/g BW daily) was sufficient to restore normal serum TSH as well as normal TRH transcript levels in the PVN. In contrast, after hypothyroid MCT8-null mice were injected with the same dose of T3 serum, TSH levels and TRH hybridization signals were not decreased at all, although serum T3 levels were in the normal range. Only treatment of hypothyroid animals with a 5 -fold higher dose of T3 $(25 \mathrm{ng} / \mathrm{g}$ BW per day, which resulted in a 5- to 6-fold elevation in T3 serum levels) effectively suppressed TSH serum levels in MCT8-null mice but not the expression of TRH mRNA in the PVN, most likely due to the impaired entry of T3 into the brain in MCT8-deficient animals.

\section{Discussion}

In light of the severe X-linked psychomotor retardation of patients with an inactivated MCT8 gene, it was surprising to realize that MCT8-null mice are born at the expected Mendelian frequency and are phenotypically indistinguishable from their wild-type littermates. The absence of obvious neurological deficits suggests a normal CNS development, and gross histological examination did not reveal any overt abnormalities.

Although unexpected, this observation is not too surprising, since with regard to brain development, genetically engineered mice have rarely reproduced all or even most of the features observed in the corresponding human conditions (20). The discrepancy between MCT8-deficient patients and MCT8-null mice is most likely explained by the existence of other thyroid hormone transporters in mice but not in humans that compensate for the absence of MCT8 in the animals. Indeed, more thyroid hormone-transporting proteins have been identified in rodents than in humans (21). For example, there is no human ortholog for the OATP1A4, which has been shown to be localized in murine neurons and to transport iodothyronines among other substrates (22, 23). It will therefore be important to identify the thyroid hormone transporters that can compensate, at least partially, for the absence of MCT8 in mice. As an alternative explanation for the differences in the neurological phenotypes of mice and humans, one could speculate that in addition to thyroid hormone, MCT8 also facilitates the transport of other molecules that are important for brain development in humans but not in mice.

Since ISH analysis revealed high expression levels of MCT8 in certain areas of the CNS as well as in peripheral organs, one could expect that T3 uptake and/or clearance might be partially impaired in MCT8-deficient animals. This assumption is supported by in vitro studies demonstrating that expression of a nonfunctional MCT8 results in reduced T3 uptake and subsequent metabolism (24). Hence, tissues in which MCT8 plays a prominent role in cellular T3 uptake may be severely hypothyroid. However, since patients with MCT8 mutations exhibit high serum T3 levels, one could assume a thyrotoxic situation in cells that express other thyroid hormone transporters. Therefore, it 
was of special interest to study in detail the tissue-specific effects of MCT8 deficiency in the mutant animals.

Interestingly, analysis of the serum thyroid hormone levels revealed that the absence of MCT8 in mice perfectly reflects the situation in patients with MCT8 mutations. Compared with those in control mice, the serum T3 levels in MCT8-null animals were increased (to 205\%) and were similar to those in the patients. Likewise, the serum T4 levels were considerably reduced in patients with MCT8 mutations, to about $60 \%$ of the mean values found in healthy subjects, and were reduced even more in MCT8-null mice, to $34 \%$ of control values. These changes in thyroid hormone levels provide evidence that the imbalance of the thyroid hormone levels in patients with MCT8 mutations is a direct consequence of the nonfunctional MCT8 transporter.

Intriguing information about the transport processes in which MCT8 is critically involved were obtained by determining the tissue-specific uptake of ${ }^{125}$ I-labeled T4 or T3 in MCT8-deficient animals. Neither entry of T3 nor of T4 into the liver was found to be affected by the absence of MCT8 - not an unexpected finding, since the liver is well known to express a variety of different thyroid hormone transporters (18). Consequently, in the liver, high serum T3 levels are fully translated into high intracellular T3 levels, as demonstrated by the increased expression levels of the hepatic T3-regulated genes $D 1$ and GPD2. Since the serum levels of the T3-regulated sex hormone-binding globulin $(S H B G)$ are elevated in patients with MCT8 mutations (25), it seems reasonable to conclude that the livers of MCT8-deficient mice and humans are in a hyperthyroid state.

Whereas hepatic thyroid hormone transport processes appear not to depend on the presence of MCT8, striking abnormalities were found in the MCT8-null mice with regard to thyroid hormone passages into the brain. In agreement with previous data (26-29), not only T4 but also T3 rapidly entered the brain in wildtype animals, putting into question the view that $\mathrm{T} 4$ is the predominant iodothyronine taken up by the CNS. Most intriguingly, uptake of T3 into the brain was almost absent in MCT8-null mice, pointing to a pivotal role of MCT8 for transporting T3 into the brain. Not too surprisingly, passage of T4 into the brain was not affected by the absence of MCT8, a finding that can be explained by the presence of other thyroid hormone transporters such as OATP1C1 at the blood brain barrier that exhibit a restricted specificity toward T4 (30).

The impaired entry of $\mathrm{T} 3$ into the brain had only mild implications for the CNS, at least in mice. As a compensatory mechanism, T3 production within this tissue was stimulated by increased D2 activities in astrocytes, and inactivation of $\mathrm{T} 3$ was reduced by decreased D3 activities in neurons. Though normal T3 values were not reached, the amount of locally produced $\mathrm{T} 3$ was still sufficient to allow a normal differentiation pattern of cerebellar neurons that did not exhibit any histological signs of a developmental delay, a hallmark of a hypothyroid situation. In the striatum, however, a mild neuronal hypothyroidism could be detected, since expression of $R C 3$, a gene well known to be regulated by thyroid hormone in this brain area (19), was slightly but significantly decreased in MCT8 mutants compared with wild-type littermates. These changes might be due to a partial reduction in neuronal $\mathrm{T} 3$ uptake in the absence of MCT8 or, alternatively, might be the consequence of an insufficient local T3 production.

A rather pronounced hypothyroid situation was observed in hypothalamic PVN neurons, where TRH transcript levels were strongly elevated in MCT8-null mice to levels comparable to those observed in athyroid Pax8 $/ /$ mice. Remarkably, the increased TRH expression could be suppressed not only in $\mathrm{Pax}^{-/-}$mice but also in MCT8-null animals by injection of a high dose of T4, indicating that the PVN neurons in general still respond to locally produced T3. However, peripheral injection of a supraphysiological dose of T3 did not affect TRH expression in MCT8-null mice, while hypothyroid wild-type animals rapidly responded to even a low dose of T3. This observation indicates that the hypothalamic TRH expression in wild-type animals is not only dependent on $\mathrm{T} 4$ supply but is also influenced by the serum T3 levels. Based on these findings, we assume that the increase in TRH expression in the PVN of MCT8-null mice may not only reflect insufficient local T4-to-T3 conversion but might also indicate an impaired uptake of T3 from the circulation, which may well represent the predominant mechanism underlying the central resistance to T3 of MCT8-null mice. In accordance with this notion, Fliers and colleagues (31) recently reported a new model for thyroid hormone feedback in the human hypothalamus, proposing tanycytes as the major cell type that provides the TRHergic neurons with T3. Since in humans and in mice, MCT8 is indeed expressed in these specialized glial cells, reduced uptake of T3 by these cells may well contribute to the observed hypothalamic phenotype.

In comparison to the hypothyroidism assessed in the hypothalamus, the pituitary of mutant animals was found to be in a euthyroid state. Neither the transcript levels of the T3-regulated genes TRH-R1 or TSH nor serum TSH levels were altered in MCT8-null mice. Only the slightly increased D2 activities in pituitary homogenates reflected a moderate hypothyroidism that might well have been compensated for by the locally increased T3 production. Remarkably, the pituitary of MCT8-null mice appears not to have sensed adequately the high serum T3 levels. This resistance to T3 was further evaluated by a TSH suppression test. For that purpose, animals were put on MMI treatment until the serum $\mathrm{T} 4$ and $\mathrm{T} 3$ levels were at the detection limit and TSH levels were consequently markedly increased. While a low dose of T3 was sufficient to suppress serum TSH levels in wildtype animals, it did not affect TSH levels in MCT8-null mice. A similar finding has been described in one patient with an MCT8 mutation in which only $\mathrm{T} 4$ but not $\mathrm{T} 3$ treatment resulted in suppressed TSH levels (32). The mechanisms underlying this T3 insensitivity are still unclear.

Taken together, our data clearly demonstrate that despite their inconspicuous phenotypical appearance, MCT8-deficient mice exhibit pronounced abnormalities in thyroid hormone metabolism. Like patients with MCT8 mutations, MCT8-null mice displayed a considerably disturbed thyroid state, with markedly increased serum T3 and decreased serum T4 levels. In the MCT8null mouse, this condition resulted in hepatic hyperthyroidism and a rather hypothyroid situation in certain brain areas, while analysis of the pituitary revealed a euthyroid state. Most strikingly, entry of T3 into the brain was completely blocked, underscoring the physiological role of MCT8 as an important T3 transporter in the CNS. Moreover, the impaired uptake of T3 into the brain might even represent the primary event that leads to the abnormal thyroid hormone levels, since an inhibited passage of $\mathrm{T} 3$ into the brain may interfere with $\mathrm{T} 3$ inactivation by neuronally localized D3, with the consequence that $\mathrm{T} 3$ accumulates in the circulation. Increased serum T3 levels in turn will stimulate D1 expression in peripheral tissues such as the liver, hence initi- 
ating a vicious circle in which consumption of $\mathrm{T} 4$ and generation of T3 are constantly increased.

Though our mouse model replicates the human phenotype with regard to the abnormal thyroid hormone metabolism, the lack of overt neurological deficits together with a normal neuronal sensitivity to T3 in vitro, as demonstrated for cerebellar Purkinje cells, suggests that neuronal MCT8 deficiency might well be compensated for by other thyroid hormone transporters more prominent in mice than in humans, thereby protecting MCT8-null mice from the neurological deficits observed in humans. Therefore, it will be most important to identify these yet-unknown transporters in order to establish suitable animal models for the human disorder. Thus, the challenge is still ahead to unravel the exact mechanisms underlying this severe human syndrome of psychomotor retardation.

\section{Methods}

Animals. Female $\mathrm{MCT}^{+/-}$mice were obtained from Deltagen. These animals were generated by homologous recombination using a targeting vector in which a 33-bp fragment of exon 2, corresponding to position nt 758-790 of the MCT8 cDNA clone (GenBank accession number NM_009197), was replaced by an IRES-lacZ Neo insert containing a polyadenylation signal at the $3^{\prime}$ end. The MCT8-null animals were bred into 2 different genetic backgrounds, C57BL/N6 and NMRI. The offspring were genotyped by PCR $\left(35\right.$ cycles at $94^{\circ} \mathrm{C}$ for 1 minute, $59^{\circ} \mathrm{C}$ for 1 minute, and $72^{\circ} \mathrm{C}$ for 1 minute) using primer GS (endogenous primer [E]) $\left(5^{\prime}\right.$ TGTGAGTATATTCACTGACCGTTTG-3'), primer GS (targeted [T], E) (5'-CAATTCAATGGTCAAAGCAGGACTG-3') and primer Neo (T) (5'-GGGCCAGCTCATTCCTCCCACTCAT- 3 ') to detect the targeted and the wild-type allele, respectively (expected PCR product size: $595 \mathrm{bp}$ for the targeted and $408 \mathrm{bp}$ for the endogenous allele). $\mathrm{Pax} 8^{-/-}$mice were obtained by mating $\mathrm{Pax}^{+/-}$male and female animals on a C57BL/N6 background; genotyping was performed by PCR analysis as described by Flamant et al. (33). Animal studies were approved by the Animal Welfare Committee of the Medizinische Hochschule Hannover, Germany. Standard laboratory chow and water were provided ad libitum. A temperature of $20^{\circ} \mathrm{C}$ and alternating 12-hour light, 12 -hour dark cycles were controlled automatically. Thyroid hormone treatment was performed by daily s.c. injection of T4 (200 ng/g BW per day) between P18 and P20, with consecutive tissue collection at P21. For TSH suppression tests, adult MCT8-null mice and wild-type littermates were kept on $0.1 \% \mathrm{MMI} / 1 \%$ sodium perchlorate in the drinking water for 12-14 weeks until T4 and T3 serum levels were close to the detection limit. Animals were injected daily for 3 consecutive days either with a single dose of $5 \mathrm{ng} / \mathrm{g}$ BW T3 (low dose) or, alternatively, with a single dose of $25 \mathrm{ng} / \mathrm{g}$ BW T3 (high dose). The animals were killed 12 hours after the last injections. Trunk blood was collected, and serum was obtained by centrifugation. All tissue and serum samples were stored at $-80^{\circ} \mathrm{C}$ before further processing.

For uptake analysis, adult MCT8-null mice and wild-type littermates (25-30 g; 4 animals each per genotype and time point) were s.c. injected with $1.13 \mu \mathrm{Ci}\left[{ }^{125} \mathrm{I}\right] \mathrm{T} 3$ (specific activity: $97.3 \mathrm{Ci} / \mathrm{mmol}$; PerkinElmer) or with $1.2 \mu \mathrm{Ci}\left[{ }^{125} \mathrm{I}\right] \mathrm{T} 4$ (specific activity: $116 \mathrm{Ci} / \mathrm{mmol}$; PerkinElmer) dissolved in 50\% 1-propanol. After the collection of $100 \mu \mathrm{l} \mathrm{blood,} \mathrm{animals}$ were perfused transcardially with ice-cold $0.9 \% \mathrm{NaCl}$. To determine the amount of radioactivity, tissues and blood samples were measured in an automatic gamma counter.

For ISH, tissue samples were embedded in Tissue-Tek medium (Sakura) and frozen in isopentane cooled on dry ice. Twenty-micrometer-thick sections of brain, liver, kidney or 16- $\mu \mathrm{m}$ sections of pituitary were cut on a cryostat (Leica Microsystems), thaw-mounted on silane-treated slides, and stored at $-80^{\circ} \mathrm{C}$. For vibratome sectioning, animals at P12 were anesthetized with isoflurane and then perfused transcardially with $4 \%$ paraformaldehyde in PBS.

Probe synthesis. A cDNA fragment corresponding to nt 1,251-1,876 of mouse MCT8 cDNA (GenBank accession number AF045692), and a fragment corresponding to nt $40-1,055$ of mouse D1 cDNA (GenBank accession number NM_007860) were generated by PCR and subcloned into the pGEM-T Easy Vector (Promega). To detect $R C 3$ and TRH transcripts, PCR fragments corresponding to nt 132-731 of the mouse RC3 cDNA (GenBank accession number NM_022029) and corresponding to nt 202-733 of the mouse TRH cDNA (GenBank accession number NM_009426) were used to prepare ${ }^{35}$ S-labeled riboprobes as described previously (34). After synthesis, the probes were subjected to mild alkaline hydrolysis for calculated time periods, as reported by Schäfer and Day (35), to generate fragments of about $250 \mathrm{nt}$.

ISH. ISH was carried out as published elsewhere (34). ${ }^{35} \mathrm{~S}$-labeled riboprobes were diluted in hybridization buffer to a final concentration of $2.5 \times 10^{4} \mathrm{cpm} / \mu \mathrm{l}$. Prior to application, radioactively labeled riboprobes for TRH and RC3 were diluted with unlabeled riboprobes at a concentration of $0.1 \mathrm{ng} / \mu \mathrm{l}$ and $0.5 \mathrm{ng} / \mu \mathrm{l}$ hybridization buffer, respectively. Following hybridization, sections were dipped in Kodak NTB2 nuclear emulsion and stored at $4^{\circ} \mathrm{C}$ for 6 days (for TRH and RC3) or for 7 days (for D1 and MCT8). Autoradiograms were developed and analyzed under dark-field illumination. For bright-field illumination, some sections were counterstained with cresyl violet.

Real-time RT-PCR. Total RNA was isolated from 3 pooled mouse livers or pituitaries per genotype using the Absolutely RNA Microprep Kit (Stratagene). The generation of cDNA and quantitative real-time PCR were performed as previously described (36). Cyclophilin was used as a housekeeping gene for normalization. The following primers were chosen to generate PCR-fragments: cyclophilin, 5'-GCAAGGATGGCAAGGATTGA-3' and 5'-AGCAATTCTGCCTGGATAGC-3'; D1, 5' -CGTGACTCCTGAAGATGATG-3' and 5'-CCAATGCCTATGGTTCCTAC-3'; D2, 5'-GGAACAGCTTCCTCCTAGAT-3' and 5'-GGTCTTCTCCGAGGCATAAT-3'; GH, 5'-CGCTTCTCGCTGCTGCTCAT-3' and 5'GTCCGAGGTGCCGAACATCA-3'; GPD2, 5'-GGCAGAAGATACCGTGAATG-3' and 5'-CTGGACAAGCCTGATGTAGA-3'; NTCP, 5'-GCATTGAGGCTCTGGCCATC-3' and 5'-AGCTGGAGCAGGTGGTCATC-3'; TRH-R1, 5'-AACTGCCGCTCTGAAGACTG-3' and 5'-ACTCTGTCACGCTGCCTGTT-3'; TSH, 5'-GTTCTGACAGCCTCGGTAT-3' and 5'-CCGCACCATGTTACTCCTTA-3'; OATP1A1, 5'-CTTACGAGTGTGCTCCAGAT-3' and 5'-GGCATACTGGAGGCAAGCTA-3'; OATP1A4, 5'-GAGCCATAGAGCACATGACT-3' and $5^{\prime}$-TTCTCCTGCCATGTTGATCC-3'; OATP1B2, 5'-GGAAGGCATAGGCGGTCATT-3' and 5'-TCGCAGTGCCTTGTCTTCTC-3'.

Pooled cerebellar cell cultures. Pooled cerebellar cultures from newborn mice were prepared and cultured with or without $1 \mathrm{nM} \mathrm{T} 3$ as described previously (37). After 14 days in vitro, cultures were fixed with $4 \%$ paraformaldehyde for 60 minutes at room temperature and immunostained with a rabbit polyclonal antibody against calbindin D28k (1:2,000; Swant) followed by incubation with an Alexa Fluor 555-labeled goat anti-rabbit antibody (1:500; Invitrogen). Calbindin-positive Purkinje cells were randomly selected ( 12 cells per well, at least 3 wells per treatment and culture) and photographed $(\times 63)$ with a DP50 camera on an AX70 Olympus microscope. Dendritic parameters were determined with the help of the NIH Image program (http://rsb.info.nih.gov/nih-image/) as described previously (37). All experiments were performed at least 3 times.

Histology. To assess the state of cerebellar development in MCT8-null animals, perfusion-fixed cerebella were cut on a vibratome and stained with an anti-calbindin antibody as described above. In addition, sections were treated with Hoechst 33258 dye ( $0.5 \mathrm{ng} / \mathrm{ml}$ for 10 minutes) in order to visu- 
alize cell nuclei. LacZ-expressing cells were identified by the detection of $\beta$-galactosidase activities on frozen sections as described previously (38).

Measurements of serum hormones and tissue of deiodinase activities. Serum T4 and $\mathrm{T} 3$ were determined by radioimmunoassay as previously described in detail (39). Brain T4 and T3 content was measured following extraction of the tissues as described in detail (40). Serum TSH levels were determined as described (38). Activities of D1 in the liver, of D2 in pituitary and brain, and of D3 in the brain were assessed as reported recently (39).

Statistics. Statistical significance between groups was determined by the 2 -tailed Student's $t$ test. A value of $P$ less than 0.05 was considered significant.

Note added in revision. During the preparation of the revised manuscript, Dumitrescu et al. (41) reported the generation and analysis of another MCT8-deficient mouse line that exhibits the same abnormal thyroid hormone parameters as described in our model.

\section{Acknowledgments}

We thank Melanie Kraus and Sabine Landmann for excellent technical assistance; Hans van Toor for performing the T4 and T3 measurements; Ellen Kaptein for determining deiodinase activities; Valerie Ashe for linguistic help; and the American Thyroid Association for generous financial support to $\mathrm{H}$. Heuer.

Received for publication February 16, 2006, and accepted in revised form January 2, 2007.

Address correspondence to: Heike Heuer, Leibniz Institute for Age Research/Fritz Lipmann Institute (FLI), Beutenbergstr. 11, D-07745 Jena, Germany. Phone: 49-3641-656021; Fax: 49-3641656040; E-mail: hheuer@fli-leibniz.de.
1. Porterfield, S.P., and Hendrich, C.E. 1993. The role of thyroid hormones in prenatal and neonatal neurological development - current perspectives. Endocr. Rev. 14:94-106.

2. Oppenheimer, J.H., and Schwartz, H.L. 1997. Molecular basis of thyroid hormone-dependent brain development. Endocr. Rev. 18:462-475.

3. de Escobar, G.M., Obregon, M.J., and del Rey, F.E. 2004. Maternal thyroid hormones early in pregnancy and fetal brain development. Best Pract. Res. Clin. Endocrinol. Metabol. 18:225-248.

4. Jones, S.A., Thoemke, K.R., and Anderson, G.W. 2005. The role of thyroid hormone in fetal and neonatal brain development. Current Opinion in Endocrinology and Diabetes. 12:10-16.

5. Bernal, J. 2005. Thyroid hormones and brain development. Vitam. Horm. 71:95-122.

6. Yen, P.M. 2001. Physiological and molecular basis of thyroid hormone action. Physiol. Rev. 81:1097-1142.

7. Bianco, A.C., Salvatore, D., Gereben, B., Berry, M.J., and Larsen, P.R. 2002. Biochemistry, cellular and molecular biology, and physiological roles of the iodothyronine selenodeiodinases. Endocr. Rev. 23:38-89.

8. Kohrle, J. 2002. Iodothyronine deiodinases. Methods Enzymol. 347:125-167.

9. Hennemann, G., et al. 2001. Plasma membrane transport of thyroid hormones and its role in thyroid hormone metabolism and bioavailability. Endocr. Rev. 22:451-476.

10. Friesema, E.C., et al. 2003. Identification of monocarboxylate transporter 8 as a specific thyroid hormone transporter. J. Biol. Chem. 278:40128-40135.

11. Friesema, E.C., et al. 2004. Association between mutations in a thyroid hormone transporter and severe X-linked psychomotor retardation. Lancet. 364:1435-1437.

12. Dumitrescu, A.M., Liao, X.H., Best, T.B., Brockmann, K., and Refetoff, S. 2004. A novel syndrome combining thyroid and neurological abnormalities is associated with mutations in a monocarboxylate transporter gene. Am. J. Hum. Genet. 74:168-175.

13. Schwartz, C.E., et al. 2005. Allan-Herndon-Dudley syndrome and the monocarboxylate transporter 8 (MCT8) gene. Am. J. Hum. Genet. 77:41-53.

14. Heuer, H., et al. 2005. The monocarboxylate transporter 8 linked to human psychomotor retardation is highly expressed in thyroid hormone-sensitive neuron populations. Endocrinology. 146:1701-1706.

15. Brockmann, K., Dumitrescu, A.M., Best, T.T., Hanefeld, F., and Refetoff, S. 2005. X-linked paroxysmal dyskinesia and severe global retardation caused by defective MCT8 gene. J. Neurol. 252:663-666.

16. Gong, D.W., Bi, S., Weintraub, B.D., and Reitman, M. 1998. Rat mitochondrial glycerol-3-phosphate dehydrogenase gene: multiple promoters, high levels in brown adipose tissue, and tissue-specific regulation by thyroid hormone. DNA Cell Biol. 17:301-309.

17. Mansouri, A., Chowdhury, K., and Gruss, P. 1998. Follicular cells of the thyroid gland require Pax8 gene function. Nat. Genet. 19:87-90.

18. Friesema, E.C., Jansen, J., Milici, C., and Visser, T.J. 2005. Thyroid hormone transporters. Vitam. Horm. 70:137-167.

19. Iniguez, M.A., et al. 1996. Cell-specific effects of thyroid hormone on RC3/neurogranin expression in rat brain. Endocrinology. 137:1032-1041.

20. Watase, K., and Zoghbi, H.Y. 2003. Modelling brain diseases in mice: the challenges of design and analysis. Nat. Rev. Genet. 4:296-307.

21. Friesema, E.C., Jansen, J., and Visser, T.J. 2005. Thyroid hormone transporters. Biochem. Soc. Trans. 33:228-232.

22. Abe, T., et al. 1998. Molecular characterization and tissue distribution of a new organic anion transporter subtype (oatp3) that transports thyroid hormones and taurocholate and comparison with oatp2. J. Biol. Chem. 273:22395-22401.

23. Abe, T., Suzuki, T., Unno, M., Tokui, T., and Ito, S. 2002. Thyroid hormone transporters: recent advances. Trends Endocrinol. Metab. 13:215-220.

24. Jansen, J., Friesema, E.C., Milici, C., and Visser, T.J. 2005. Thyroid hormone transporters in health and disease. Thyroid. 15:757-768.

25. Herzovich, V., et al. 2006. Unexpected peripheral markers of thyroid function in a patient with a novel mutation of the MCT8 thyroid hormone transporter gene. Horm. Res. 67:1-6.

26. Dratman, M.B., and Crutchfield, F.L. 1989. Thyroxine, triiodothyronine, and reverse triiodothyronine processing in the cerebellum: autoradiographic studies in adult rats. Endocrinology. 125:1723-1733.

27. Dratman, M.B., Crutchfield, F.L., and Schoenhoff, M.B. 1991. Transport of iodothyronines from bloodstream to brain: contributions by blood: brain and choroid plexus:cerebrospinal fluid barriers. Brain Res. 554:229-236.

28. Cheng, L.Y., et al. 1994. Film autoradiography identifies unique features of [125I]3,3' $5^{\prime}$-(reverse) triiodothyronine transport from blood to brain. J. Neurophysiol. 72:380-391.

29. Farwell, A.P., Dubord-Tomasetti, S.A., Pietrzykow- ski, A.Z., and Leonard, J.L. 2006. Dynamic nongenomic actions of thyroid hormone in the developing rat brain. Endocrinology. 147:2567-2574.

30. Sugiyama, D., et al. 2003. Functional characterization of rat brain-specific organic anion transporter (Oatp14) at the blood-brain barrier: high affinity transporter for thyroxine. J. Biol. Chem. 278:43489-43495.

31. Alkemade, A., et al. 2006. Novel neuroanatomical pathways for thyroid hormone action in the human anterior pituitary. Eur. J. Endocrinol. 154:491-500.

32. Biebermann, H., et al. 2005. Extended clinical phenotype, endocrine investigations and functional studies of a loss-of-function mutation A150V in the thyroid hormone specific transporter MCT8. Eur. J. Endocrinol. 153:359-366.

33. Flamant, F., et al. 2002. Congenital hypothyroid Pax8(-/-) mutant mice can be rescued by inactivating the TRalpha gene. Mol. Endocrinol. 16:24-32.

34. Heuer, H., Schafer, M.K., O’Donnell, D., Walker, P., and Bauer, K. 2000. Expression of thyrotropin-releasing hormone receptor 2 (TRH-R2) in the central nervous system of rats. J. Comp. Neurol. 428:319-336.

35. Schäfer, M.K.H., and Day, R. 1995. In situ hybridization techniques to study processing enzyme expression at the cellular level. Methods Neurosci. 23:16-44.

36. Mittag, J., et al. 2005. Athyroid Pax8-/- mice cannot be rescued by the inactivation of thyroid hormone receptor alpha1. Endocrinology. 146:3179-3184.

37. Heuer, H., and Mason, C.A. 2003. Thyroid hormone induces cerebellar Purkinje cell development via the thyroid hormone receptor alpha1. J. Neurosci. 23:10604-10612.

38. Rabeler, R., et al. 2004. Generation of thyrotropinreleasing hormone receptor 1 -deficient mice as an animal model of central hypothyroidism. Mol. Endocrinol. 18:1450-1460.

39. Friedrichsen, S., et al. 2003. Regulation of iodothyronine deiodinases in the pax8-/- mouse model of congenital hypothyroidism. Endocrinology. 144:777-784

40. Reyns, G.E., Janssens, K.A., Buyse, J., Kuhn, E.R., and Darras, V.M. 2002. Changes in thyroid hormone levels in chicken liver during fasting and refeeding. Comp. Biochem. Physiol. B. Biochem. Mol. Biol. 132:239-245.

41. Dumitrescu, A.M., Liao, X.H., Weiss, R.E., Millen, K., and Refetoff, S. 2006. Tissue-specific thyroid hormone deprivation and excess in monocarboxylate transporter $(\mathrm{mct})$ 8-deficient mice. Endocrinology. 147:4036-4043. 